\title{
Eye Movement Signal Classification for Developing Human-Computer Interface Using Electrooculogram
}

\author{
M. Thilagaraj $\mathbb{D}^{1},{ }^{1}$ B. Dwarakanath $\mathbb{D}^{\circ},{ }^{2}$ S. Ramkumar $\left(\mathbb{D},{ }^{3}\right.$ K. Karthikeyan $\left(\mathbb{D},{ }^{4}\right.$ A. Prabhu $(\mathbb{D}){ }^{5}$ \\ Gurusamy Saravanakumar $\mathbb{D},{ }^{6}$ M. Pallikonda Rajasekaran $\mathbb{D D}^{7}$ and N. Arunkumar $\mathbb{D}^{8}$ \\ ${ }^{1}$ Department of Electronics and Instrumentation Engineering, Karpagam College of Engineering, Coimbatore, Tamil Nadu, India \\ ${ }^{2}$ Department of Information Technology, SRM Institute of Science and Technology, Chennai, Tamil Nadu, India \\ ${ }^{3}$ School of Computing, Kalasalingam Academy of Research and Education, Krishnankoil, Virudhunagar, Tamil Nadu, India \\ ${ }^{4}$ Department of Electrical and Electronics Engineering, Ramco Institute of Technology, Rajapalayam, Tamil Nadu, India \\ ${ }^{5}$ K. Ramakrishnan College of Engineering, Trichy, Tamil Nadu, India \\ ${ }^{6}$ Department of Electrical and Electronics Technology, Ethiopian Technical University, Addis Ababa, Ethiopia \\ ${ }^{7}$ Department of Electronics and Communication Engineering, Kalasalingam Academy of Research and Education, Krishnankoil, \\ Virudhunagar, Tamil Nadu, India \\ ${ }^{8}$ Department of Biomedical Engineering, Rathinam Technical Campus, Coimbatore, Tamil Nadu, India
}

Correspondence should be addressed to Gurusamy Saravanakumar; saravanakumar.gurusamy@etu.edu.et

Received 31 October 2021; Accepted 18 November 2021; Published 8 December 2021

Academic Editor: Bhagyaveni M.A

Copyright (c) 2021 M. Thilagaraj et al. This is an open access article distributed under the Creative Commons Attribution License, which permits unrestricted use, distribution, and reproduction in any medium, provided the original work is properly cited.

\begin{abstract}
Human-computer interfaces (HCI) allow people to control electronic devices, such as computers, mouses, wheelchairs, and keyboards, by bypassing the biochannel without using motor nervous system signals. These signals permit communication between people and electronic-controllable devices. This communication is due to HCI, which facilitates lives of paralyzed patients who do not have any problems with their cognitive functioning. The major plan of this study is to test out the feasibility of nine states of HCI by using modern techniques to overcome the problem faced by the paralyzed. Analog Digital Instrument T26 with a five-electrode system was used in this method. Voluntarily twenty subjects participated in this study. The extracted signals were preprocessed by applying notch filter with a range of $50 \mathrm{~Hz}$ to remove the external interferences; the features were extracted by applying convolution theorem. Afterwards, extracted features were classified using Elman and distributed time delay neural network. Average classification accuracy with $90.82 \%$ and $90.56 \%$ was achieved using two network models. The accuracy of the classifier was analyzed by single-trial analysis and performances of the classifier were observed using bit transfer rate (BTR) for twenty subjects to check the feasibility of designing the HCI. The achieved results showed that the ERNN model has a greater potential to classify, identify, and recognize the EOG signal compared with distributed time delay network for most of the subjects. The control signal generated by classifiers was applied as control signals to navigate the assistive devices such as mouse, keyboard, and wheelchair activities for disabled people.
\end{abstract}

\section{Introduction}

Healthy human beings use their muscles to move, drive vehicles, and move objects. The muscles receive commands from the brain. Certain neuromuscular disorders impair the communication between the brain and the muscles, causing partial or total paralysis depending on the severity of disorders. In most cases, eye muscle functions are not initially affected. These disorders do not impair cognitive abilities, and such individuals are aware of their environment, creating a locked-in state. Medical research helps such individuals live longer, and hence rehabilitation becomes essential to improve their lifestyle and reduce the burden on their caregivers. The human-computer interface provides a digital communication channel for paralyzed people by assisting human cognitive or motor neuron function through bypassing the biological channel communication. Some of the prominent interfaces developed for impaired 
persons include smartphones, keyboards, game controllers, sleep monitoring systems, and drowsiness detection systems [1-12].

Electrooculography (EOG) principle was used widely and successfully to detect eye movements to control the human-computer interface because of its noninvasive, portable, and inexpensive activities and because it can be used in almost all environments [13]. In recent years, much research relating to electrooculography supported interface for HCI has been matured to overcome the problem that occurred due to the locked-in state and motor neuron disease. In most of the previous studies, eye blink was defined as an election command for completing the tasks, but sometimes the subject or person was unable to control the eye blinks because blinks would occur unwillingly. To avoid this condition, this paper establishes a new protocol for acquiring signals for both events and nonevents to ensure the possibilities of nine states of HCI by using different eleven eye movements. From these eleven different eye movements, eight were considered as events and three were nonevents. Eye blinks, open, and stare were considered the nonevents during this study. This research mainly focuses on checking the possibilities of design and developing nine states of HCI using classification techniques and recognition rate of $\mathrm{HCI}$ with the help of single-trial analysis and accuracy by using bit transfer rate.

Section 2 provides the background details. Section 3 explains protocol, preprocessing techniques. Section 4 details the feature extraction, Section 5 deals with classification techniques used in this investigation. Outcome analysis is presented in Section 6, and the conclusion and future study are discussed in Sections 7 and 8.

\section{Background Study}

Nowadays, electrooculography-based human-computer interaction is used by paralyzed people who have neurodegenerative problems. It acts as a good communication device for conveying their thoughts with others by using the technology. The following are some of the most prominent publications that are useful to people with disabilities: a study by Saravanakumar et al. developed EOG based keyboard system in synchronous mode and asynchronous mode using peak amplitude features and obtained the accuracy of $94.2 \%$ and $98.79 \%$ [14]. In one of the earliest studies, Barea et al. implemented a wheelchair guidance system, which uses four-state systems to guide a wheelchair to move forward, backward, right, and left. The result obtained in this study shows that weakened individuals frequently involve about $10-15 \mathrm{~min}$ to be trained to use this arrangement [15]. Thilagaraj et al. devised the assistive device for ALS and semiparalyzed persons using spectral density features with a dynamic network model from twenty individuals and obtained the accuracy of $91.95 \%$ and 90.28 [16]. Another study by Tsai et al. concentrated on an eye writing system using EOG signals of eye movements corresponding to pattern recognition of ten Arabic numerals and four mathematical operators and attained 95\% accuracy compared to other writing systems [17]. He and Li modeled the audio speller for
LIS person through EOG signals using waveform detection algorithm and SVM algorithm and obtained an accuracy of 94.40\% [18].

Jayaprbhu et al. developed aided assistive intelligence system for ALS persons using RMS features trained with PNN architecture models from 15 subjects and obtained an accuracy of $94.00 \%$ for young aged subjects, $93.27 \%$ for old aged subjects, and $90.37 \%$ for ALS-affected individuals [19]. Supratak et al. created a sleep detection system for drivers using time-invariant features with the CNN network model and obtained the accuracy of $86.20 \%$ and $73.70 \%$ for two different datasets [20]. Obeidat et al. developed a wheelchair for a paralyzed person using Bayesian Linear Discriminant Analysis and obtained an accuracy of 95\% from fourteen subjects [21]. Jayaprbhu et al. developed EEG-based BCI for ALS-affected persons using the convolution neural network and cross power spectral density for four subjects from fifteen subjects and obtained the accuracy of $91.18 \%$ and $86.88 \%$ [22]. Xiao et al. modeled four-state EEG-based BCI for SCI-affected individuals using CWT featured with a hybrid neural network and obtained an accuracy of $93.86 \%$ [23]. Kai et al. designed the rehabilitative device for LIS patients using local binary patterns features with Grey Wolf optimization algorithm and obtained $98.33 \%$ to $88.33 \%$ for the subject's age group between 20 and 60 from nine subjects [24].

Dev et al. designed a wheelchair controller for quadriplegic patients using PSD features and a fuzzy classifier and obtained good accuracy using one electrode system from NeuroSky Headset [25]. Lokman et al. created BCI to control the finger movement from thirteen subjects using genetic algorithm features with MLP and SVM classifiers and obtained the accuracy of $97.34 \%$ and $97.46 \%$ [26]. Turnip et al. modeled wheelchairs for SCI-affected individuals for four tasks and obtained 90\% accuracy using ANFIS classifiers from four subjects belonging to the age group between 25 and 26 [27]. Ilyas et al. developed BCI to determine the patterns using several classifiers and obtained the maximum accuracy of $73.03 \%$ for logistic regression and $68.97 \%$ for SVM classifiers [28]. A background study on EOG classification exposed that very limited work has been presented on identifying the EOG signals using Elman and Distributed time delay neural network, and much research focuses on conventional movements only. Through this research, we consider the possibility of recognizing eight-task (events) and three-task (nonevents) movements using feedback networks. The achievement of the feature extraction approach was compared using a feedback network to confirm the outcomes.

\section{Experimental Protocol}

We started our study with a preliminary study with two subjects, a male and a female, to determine the signals patterns. For each individual subject, the pattern generation was different, and also we identified that all the different tasks produced different patterns. Therefore, we concluded that classifying all the tasks was possible during the study.

ADT26 bioamplifier was used to gather EOG signals for 20 normal persons. Signals gathered from the participants 
were sampled at $100 \mathrm{HZ}$ and each band was split with $2 \mathrm{HZ}$ from 0.1 to $16 \mathrm{~Hz}$. The methodology of the study implemented in this research was described by the same author in his prior study $[29,30]$.

\section{Feature Extraction}

A feature extraction algorithm using convolution theorem was used to extract the prominent features from the bandpass filtered EOG signals for all eleven tasks. The theorem described that a mathematical operation on two-channel signals $X_{b}{ }^{j}$ and $R_{b}{ }^{j}$ in time domain equals pointwise multiplication in the frequency domain of the original signals converted. Finally, the convoluted signals F1 and $R_{1}$ are written as $F_{1} * R_{1}$, so that convolution operator was indicated by using $*$ symbolized:

$$
\begin{aligned}
& X_{b}^{j}=\left\{x_{b i}^{j}\right\}_{i=1,2, \ldots 100, b=1,2, \ldots, 8}, \\
& R_{b}^{j}=\left\{x_{b i}^{j}\right\}_{i=100,99, \ldots 1, b=1,2, \ldots, 8}, \\
& F_{1}=F\left\{X_{b}^{j}\right\}, \\
& R_{1}=F\left\{R_{b}^{j}\right\} .
\end{aligned}
$$

Let $\mathrm{F}$ represent the Fourier transform, so that $F\left(X_{b}^{j}\right)$ is a Fourier signal and $F\left(R_{b}^{j}\right)$ is a reverse and shifted Fourier signal of the Fourier transform of $\mathrm{F}_{1}$ and $\mathrm{R}_{1}$ correspondingly. Then,

$$
F\left\{F_{1} * R_{1}\right\}=F\left\{F_{1}\right\} \cdot F\left\{R_{1}\right\}
$$

where the dot indicates the pointwise multiplication. Thus, equation (2) can also be written as

$$
F\left\{F_{1} \cdot R_{1}\right\}=F\left\{F_{1}\right\} * F\left\{R_{1}\right\}
$$

By implementing the convolution equation, we can write down

$$
F_{1} * R_{1}=\sum_{n=0}^{N-1}\{F\{F 1\} . F\{R 1\}\} .
$$

From this feature extraction technique, 16 features were taken out for individual trial. The features were extracted for 10 such trials for each task. A neural network classifier was implemented to train and test 110 data samples for one subject. The feature sets obtained from the single trial for each task are demonstrated in Figure 1.

\section{Classification Techniques}

To categorize the signal obtained from the eye movements for 20 subjects, two neural network models were planned to recognize the eleven different tasks. Two classical networks, particularly the ERNN and DTDNN, were applied in this study. The convolution features were given as input to the following networks. Outcomes of two network models were related to validating the possibility of designing multistates HCI.
5.1. Elman Recurrent Neural Network (ERNN). ERNN has feedback connections that affix the capability to study the physical aspects of the data. The network architecture consists of a context layer, which was equal to the hidden layer in an ordinary network model to make a copy. The main aspects of this layer maintain the previous state of the hidden layer at the previous pattern arrangement. Due to this reason, the training time testing time and classification rate of the network model were higher than those of FFNN [31-34].

\subsection{Distributed Time Delay Neural Network. A DTDNN was} one of the most powerful dynamic network models, and its performance was high compared to other static network models because it has a capacity to learn time varying and also the sequential patterns due to its memory storage. The layers involved in this architecture have biases, so each layer has added the weight from the earlier layers, and the last layer was completed with output layers. It assigns the tapped delay lines throughout the network. The only difference between the DTDNN and the TDNN is that the second input argument is a cell array that contains the tapped delay to be used in each layer. It was similar to FFNN, except that each input and layer weight have a tap delay line associated with it [35-38].

During the parameters settings, we fixed the neural network classifier in the below-mentioned condition. The two network models were trained using backpropagation (Gradient Descent) for the ERNN model and Levenberg backpropagation training algorithm for the DTDNN model with eight hidden neurons, sixteen input neurons, and four output neurons. The two network models were trained with $100 \%$ data samples and trained with $75 \%$ for each individual subject, and also, we fixed testing error tolerance of 0.1 during the testing with a .001 learning rate of the network model experimentally. During the classification, we normalized the samples between zero and one using the normalization procedure. The maximum iteration of the network was fixed and limited to 1000, and network error falls below 0.001 to determine the network performance $[34,39,40]$. Figures 2 and 3 present the construction of the ERNN and DTDNN models used in this experiment.

\section{Outcome Analysis}

Tables 1 and 2 showed the average accuracy of the network models implemented in the study. The results showed that the ERNN model has the highest overall classification accuracy of $90.82 \%$, whereas the DTDNN model has the highest overall classification accuracy of $90.56 \%$. The maximum classification accuracy of $91.91 \%$ and $91.76 \%$ was observed for subject 12 . The average maximum classification accuracy of $93.97 \%$ and $3.87 \%$ was observed. The average minimum classification accuracy of $86.10 \%$ and $86.06 \%$ was observed. Average testing and training times for twenty subjects were 5.35 and 0.9205 seconds and 3.64 and 0.6545 for the convolution features using ERNN and DTDNN, respectively. From the study, we 


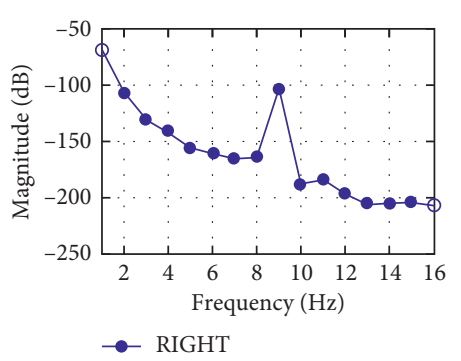

(a)

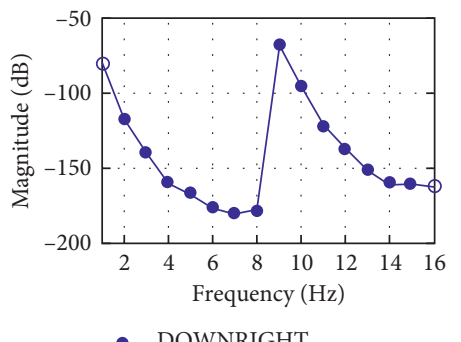

(d)

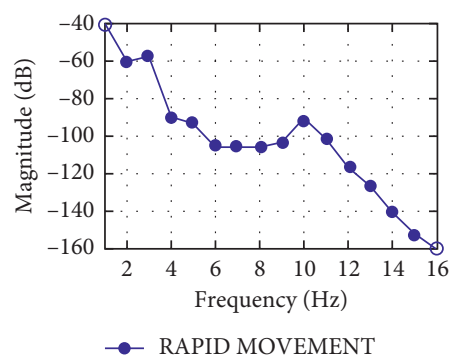

(g)

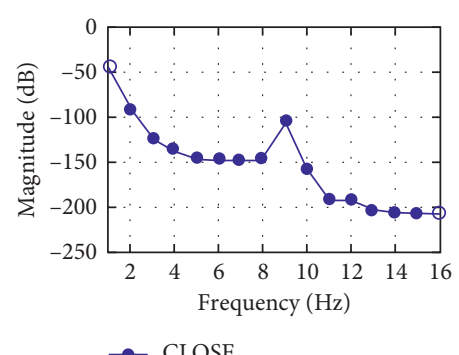

(j)

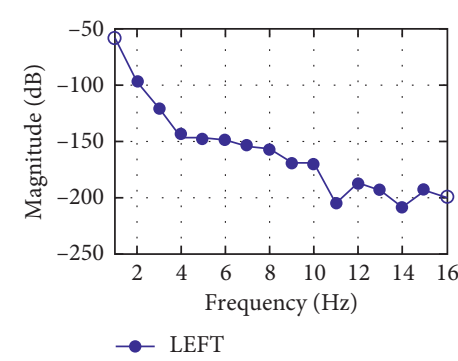

(b)

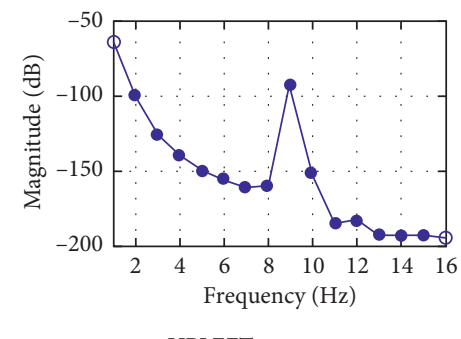

(e)

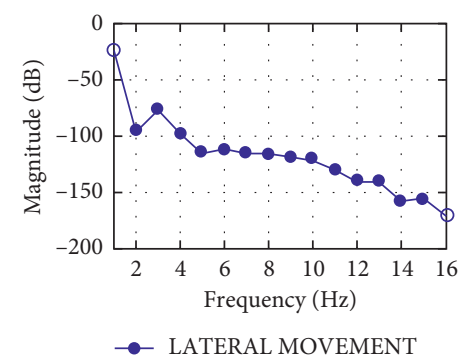

(h)

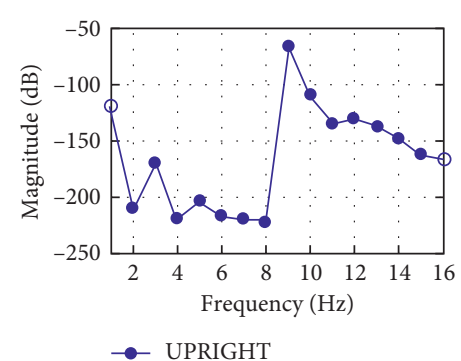

(c)

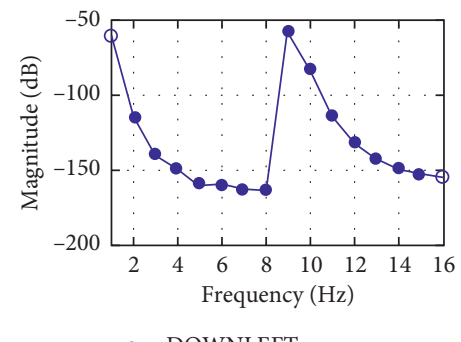

(f)

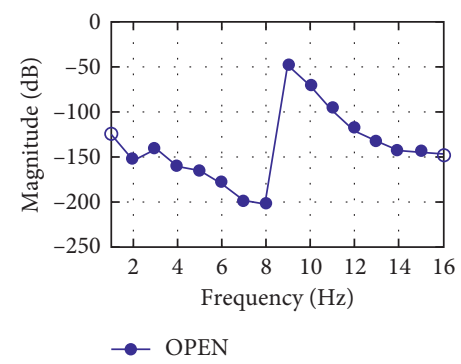

(i)

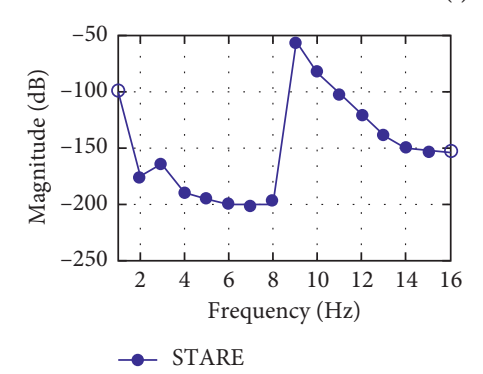

(k)

Figure 1: Signals extracted by implementing the feature extraction technique for (a) right, (b) left, (c) up right, (d) down right, (e) up left, (f) down left, (g) rapid movement, (h) lateral movement, (i) open, (j) close, and (k) stare tasks for Subject 2 using convolution theorem.

observed that the ERNN model performed better than the DTDNN model throughout the investigation, which is depicted in Figure 4. From the outcomes obtained in recognizing the eleven eye movements, it was seen that the performance of ERNN models using convolution features was high compared to the DTDNN architecture used in this study.

6.1. Single-Trial Analysis (STR) Using ERNN and DTDNN with Convolution Features. From the STR, it was concluded that $\mathrm{S} 1$ achieved $80 \%$ accuracy for rapid movement and open and $70 \%$ accuracy for left, up right, down right, up left, down left, close, and stare. S2 achieved $80 \%$ accuracy for up right, up left, and close and 70\% accuracy for right, left, down right, down left, and stare. S3 achieved $80 \%$ accuracy for left, down right, and stare and $70 \%$ accuracy for up right, down left, and open. S4 achieved only $80 \%$ accuracy for down right, down left, open, and stare and 70\% accuracy for right, up right, up left, and close. S5 achieved only $80 \%$ accuracy for down right and 70\% accuracy for left, up right, up left, open, and stare. S6 achieved only $80 \%$ accuracy for left and down right and $70 \%$ accuracy for up right, up left, and down left. S7 achieved only $80 \%$ accuracy for left, down right, and up left and 70\% accuracy for right, up right, and stare. S8 achieved $100 \%$ accuracy for close; $90 \%$ accuracy for left; $80 \%$ 


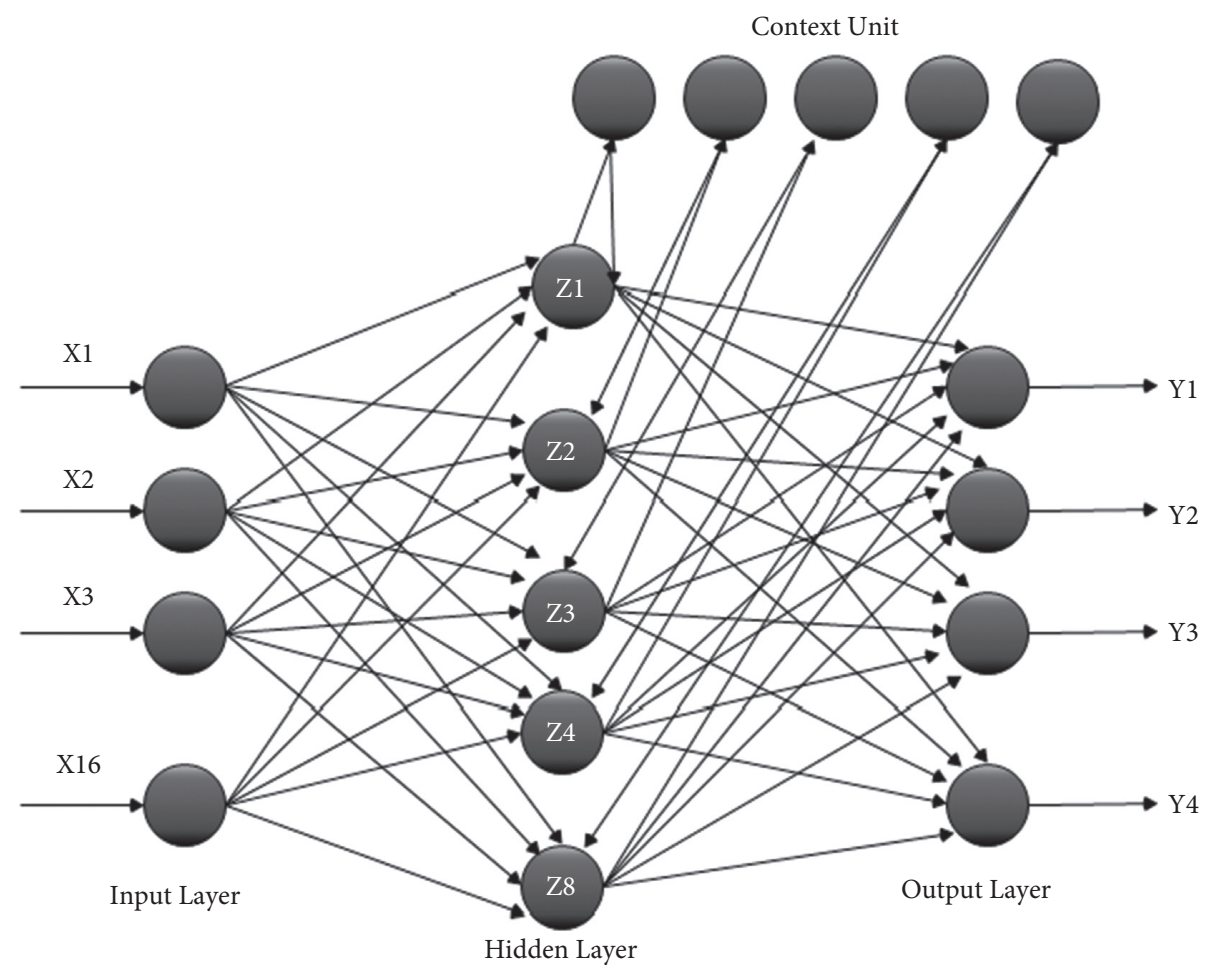

FIGURE 2: Elman recurrent neural network model.

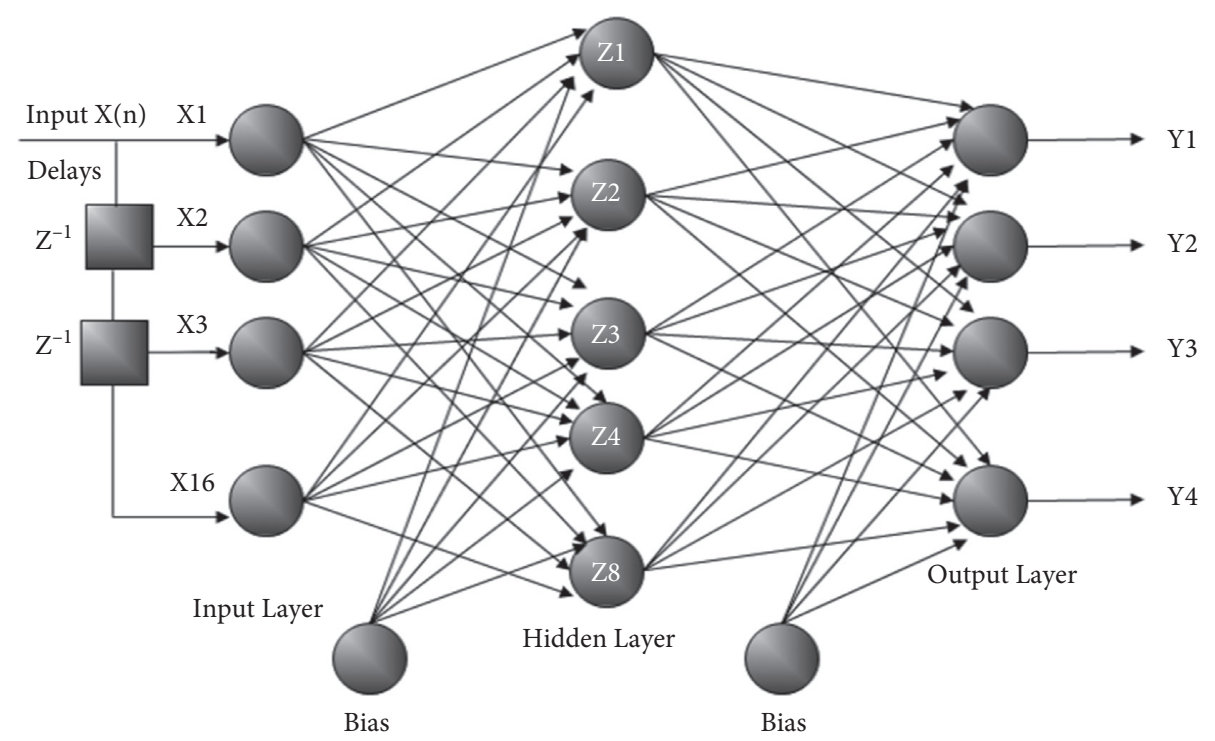

Figure 3: Distributed time delay neural network model.

accuracy for up right, up left, and down left; and 70\% accuracy for right, down right, open, and stare. S9 achieved $80 \%$ accuracy for down right and $70 \%$ accuracy for left, up left, and open. S10 achieved 100\% accuracy for left and $80 \%$ accuracy for up right and stare. S11 achieved 80\% accuracy for lateral movement and close and $70 \%$ accuracy for up right, open, and stare. S12 achieved 80\% accuracy for open and stare and $70 \%$ accuracy for left, up right, down right, up left, and down left. S13 achieved 80\% accuracy for down right and 70\% accuracy for down left and stare. S14 achieved
$80 \%$ accuracy for up right and $70 \%$ accuracy for left, up left, and stare. S15 achieved only 80\% accuracy for up left and $70 \%$ accuracy for up right, down right, and down left. S16 achieved $80 \%$ accuracy for down left and open and $70 \%$ accuracy for left, up right, and stare. S17 achieved $80 \%$ accuracy for up right and down left and 70\% accuracy for open and stare. S18 achieved 90\% accuracy for open, 80\% accuracy for left up right and stare, and 70\% accuracy for up left. S19 achieved 80\% accuracy for down right and 70\% accuracy for left, up right, open, and stare. S20 achieved $80 \%$ 
TABLe 1: Performance accuracy of convolution features using ERNN.

\begin{tabular}{|c|c|c|c|c|c|c|c|c|}
\hline \multirow{2}{*}{ S. no. } & \multirow{2}{*}{ Sub. } & \multirow{2}{*}{ Hidden neuron } & \multirow{2}{*}{ Training time (sec) } & \multirow{2}{*}{ Testing time (sec) } & \multicolumn{4}{|c|}{ Recognizing accuracy } \\
\hline & & & & & Max & Min & Mean & Std. \\
\hline 1 & S1 & 8 & 5.31 & 0.92 & 93.64 & 83.64 & 90.73 & 2.60 \\
\hline 2 & $\mathrm{~S} 2$ & 8 & 5.12 & 0.94 & 93.64 & 87.27 & 90.86 & 2.03 \\
\hline 3 & S3 & 8 & 5.38 & 0.91 & 94.55 & 85.45 & 90.94 & 2.46 \\
\hline 4 & $\mathrm{~S} 4$ & 8 & 5.43 & 0.88 & 93.64 & 86.36 & 90.90 & 2.13 \\
\hline 5 & S5 & 8 & 5.42 & 0.93 & 93.78 & 85.45 & 90.58 & 2.32 \\
\hline 6 & S6 & 8 & 5.45 & 0.84 & 93.64 & 86.36 & 90.59 & 2.23 \\
\hline 7 & S7 & 8 & 5.43 & 0.94 & 93.64 & 85.56 & 90.77 & 2.48 \\
\hline 8 & S8 & 8 & 5.32 & 0.92 & 93.64 & 86.36 & 90.90 & 2.22 \\
\hline 9 & S9 & 8 & 5.34 & 0.86 & 94.55 & 85.45 & 90.91 & 2.15 \\
\hline 10 & $\mathrm{~S} 10$ & 8 & 5.30 & 0.89 & 93.64 & 84.55 & 90.31 & 2.41 \\
\hline 11 & $\mathrm{~S} 11$ & 8 & 5.32 & 0.96 & 93.64 & 86.36 & 90.58 & 2.17 \\
\hline 12 & $\mathrm{~S} 12$ & 8 & 5.41 & 0.93 & 95.55 & 88.18 & 91.91 & 2.04 \\
\hline 13 & $\mathrm{~S} 13$ & 8 & 5.39 & 0.95 & 93.64 & 86.36 & 90.90 & 1.93 \\
\hline 14 & S14 & 8 & 5.34 & 0.92 & 93.64 & 86.36 & 91.12 & 2.12 \\
\hline 15 & S15 & 8 & 5.34 & 0.95 & 94.55 & 87.09 & 90.71 & 1.92 \\
\hline 16 & S16 & 8 & 5.43 & 0.89 & 94.55 & 85.56 & 90.77 & 2.20 \\
\hline 17 & S17 & 8 & 5.52 & 0.90 & 93.64 & 86.36 & 90.68 & 2.00 \\
\hline 18 & S18 & 8 & 5.42 & 0.92 & 93.64 & 85.56 & 90.77 & 2.22 \\
\hline 19 & S19 & 8 & 5.42 & 0.97 & 93.64 & 87.27 & 90.82 & 2.09 \\
\hline 20 & $\mathrm{~S} 20$ & 8 & 5.40 & 0.99 & 94.55 & 86.36 & 90.64 & 1.94 \\
\hline
\end{tabular}

TABLE 2: Performance accuracy of convolution features using DTDNN.

\begin{tabular}{lccccccc}
\hline \multirow{2}{*}{ S. no. } & Sub. & Hidden neuron & Mean training time $(\mathrm{sec})$ & Mean testing time (sec) & \multicolumn{3}{c}{ Recognizing accuracy } \\
& & & & & Max & Min & Mean \\
Std.
\end{tabular}

accuracy for up right and stare and 70\% accuracy for left and open using the ERNN model.

From the STR, it was concluded that S1 achieved 80\% accuracy for rapid movement and open and 70\% accuracy for left, up right, down right, up left, down left, close, and stare. S2 achieved $80 \%$ accuracy for up right, up left, and close and $70 \%$ accuracy for right, left, down right, down left, and stare. S3 achieved $80 \%$ accuracy for left, down right, and stare and $70 \%$ accuracy for up right, down left, and open. S4 achieved only $80 \%$ accuracy for down right, down left, open, and stare and $70 \%$ accuracy for right, up right, up left, and close. S5 achieved only 70\% accuracy for left, up right, up left, open, and stare. S6 achieved only $80 \%$ accuracy for left and down right and $70 \%$ accuracy for up right, up left, and down left. S7 achieved only $80 \%$ accuracy for left, down right, and up left and 70\% accuracy for right, up right, and stare. S8 achieved 100\% accuracy for close; 90\% accuracy for left; $80 \%$ accuracy for up right, up left, and down left; and $70 \%$ accuracy for right, down right, open, and stare. S9 achieved $80 \%$ accuracy for down right and $70 \%$ accuracy for left, up left, open, and stare. S10 achieved 100\% accuracy for left; $80 \%$ accuracy for up right and stare; and 70\% accuracy 


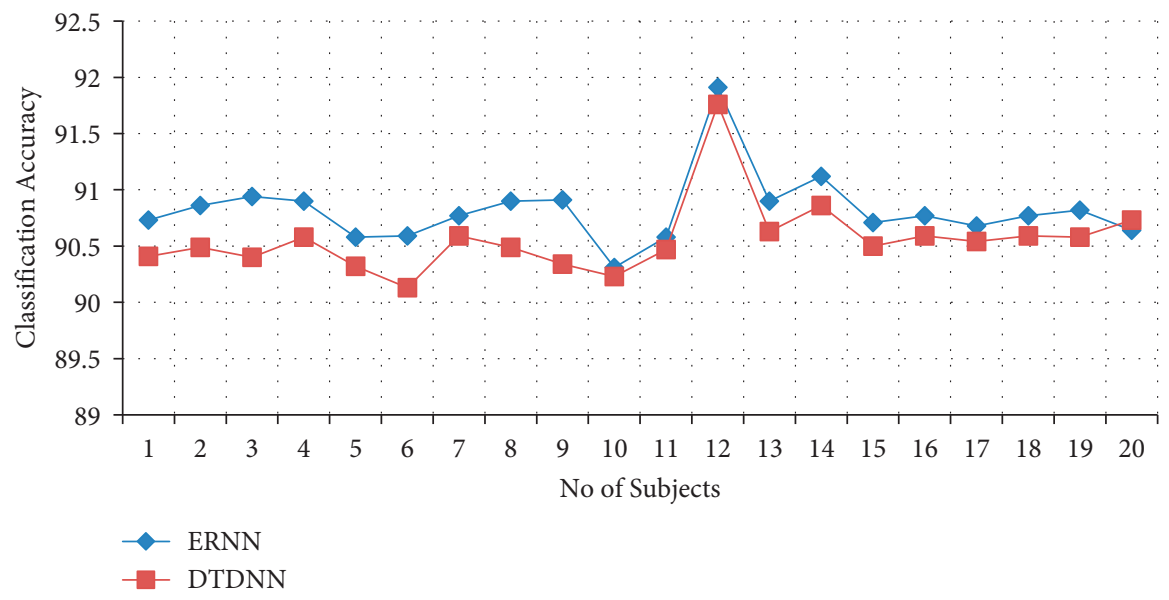

FIgURE 4: Average classification accuracy of the convolution features using ERNN and DTDNN.

for up left, open, and stare. S11 achieved 80\% accuracy for lateral movement and close and $70 \%$ accuracy for up right, open, and stare. S12 achieved 80\% accuracy for open and close and 70\% accuracy for left, up right, down right, up left, and down left. S13 achieved 80\% accuracy for down right and 70\% accuracy down left and stare. S14 achieved $80 \%$ accuracy for up right and 70\% accuracy for left, up left, and stare. S15 achieved only 80\% accuracy for up left and 70\% accuracy for up right, down right, and down left. S16 achieved $80 \%$ accuracy for down left and open and 70\% accuracy for left, up right, and stare. S17 achieved 80\% accuracy for up right and down left and 70\% accuracy for open and stare. S18 achieved $90 \%$ accuracy for open, $80 \%$ accuracy for left up right and stare, and 70\% accuracy for up left. S19 achieved 80\% accuracy for down right and 70\% accuracy for left, up right, open, and stare. S20 achieved $80 \%$ accuracy for up right and stare and $70 \%$ accuracy for left and open using the DTDNN model.

The result of the nine-state HCI system designed for each subject was explored through a single-trial analysis using ERNN and DTDNN for convolution features. From the STR, it was observed that, for subjects 7 and 8 , the accuracy rate was high at a mean of $90 \%$ for events and $85 \%$ for nonevents using convolution features, and for subject 13, the acceptance rate was low at a mean of $70 \%$ for events and $75 \%$ for nonevents using convolution features with ERNN and DTDNN network model used in this investigation. From the single-trial analysis, it was evident that eighty percentages of the signals have a recognition rate of eight and above for some subjects such as S1, S2, S3, S4, S5, S13, S19, and S20 where the recognition rates were not appreciable. From the analysis, we concluded that more training was required to improve the recognition accuracy of the events as well as nonevents. From the result, it was identified that practicability of developing a nine-state HCI is possible for some subjects participating in this experiment using convolution features for DTDNN, while for some of the subjects such as S1, S2, S3, S4, S5, S13, S19, and S20, the mean recognition accuracy of nine-state HCI was around $80 \%$ only, so the subjects involved in the study were not able to perform some of the trials correctly and feel that the task was hard to perform continuously. They were also unable to switch over from one task to another immediately.

Sensitivity and specificity were the mathematical evaluation of the classification test, where sensitivity calculates the events that were correctly identified. The sensitivity, specificity, and accuracy of the individual subject were calculated from equations (5), (6), and (7):

True positive $(\mathrm{TP})=$ correctly classified trials

False positive $(\mathrm{FP})=$ incorrectly classified trials

True negative $(\mathrm{TN})=$ correctly classified nonevent trials

False negative $(\mathrm{FN})=$ incorrectly classified nonevents trials

$$
\text { Sensitivity }=\frac{\mathrm{TP}}{(\mathrm{TP}+\mathrm{FN})}
$$

Specificity calculates the events that were identified as wrongly classified:

$$
\text { Specificity }=\frac{\mathrm{TN}}{(\mathrm{TN}+\mathrm{FP})} \text {. }
$$

6.2. Accuracy. The accuracy of the subject was measured by differentiating events and nonevents cases correctly. To calculate the accuracy test, estimate the proportion of TP and TN in all appraised cases proposed by [41, 42]. The mathematical formula to calculate the accuracy is stated as

$$
\text { Accuracy }=\frac{(\mathrm{TP}+\mathrm{TN})}{(\mathrm{TP}+\mathrm{TN}+\mathrm{FP}+\mathrm{FN})} \text {. }
$$

From the investigational result, it was analyzed that the convolution features using ERNN outperform the DTDNN model for some subjects. At the same time, the DTDNN model gave better accuracy for some subjects during this study. This confirms that EOG signals were subject variants. After comparison, it was analyzed that ERNN with convolution features was analyzed to be the best classifier model among the other network model designed for eleven tasks. 


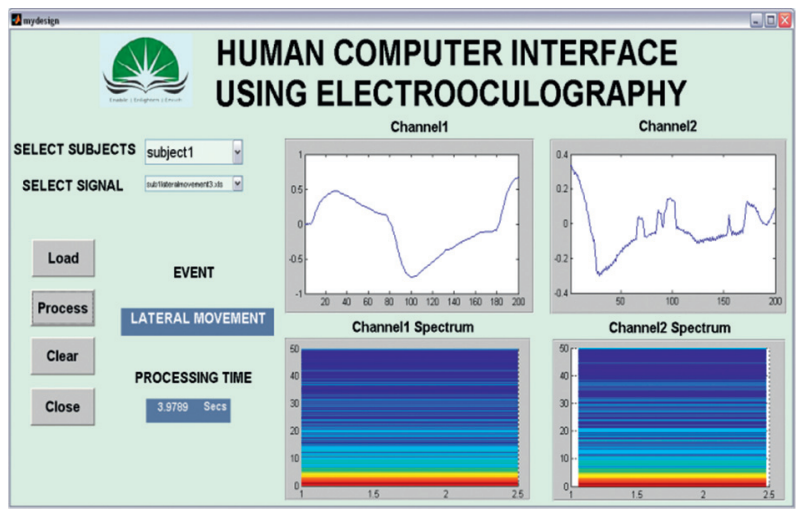

FIgURE 5: Recognizing accuracy evaluation using GUI.

TABLE 3: Bit transfer rate of ERNN using convolution features.

\begin{tabular}{|c|c|c|c|c|c|}
\hline \multirow{2}{*}{ S. no. } & \multirow{2}{*}{ Sub. } & \multicolumn{4}{|c|}{ Bit transfer rate } \\
\hline & & Maximum accuracy & Minimum accuracy & Mean accuracy & BTR accuracy \\
\hline 1 & S1 & 93.64 & 83.64 & 90.73 & 81.17 \\
\hline 2 & S2 & 93.64 & 87.27 & 90.86 & 81.43 \\
\hline 3 & S3 & 94.55 & 85.45 & 90.94 & 81.59 \\
\hline 4 & S4 & 93.64 & 86.36 & 90.90 & 81.51 \\
\hline 5 & S5 & 93.78 & 85.45 & 90.58 & 80.87 \\
\hline 6 & S6 & 93.64 & 86.36 & 90.59 & 80.89 \\
\hline 7 & S7 & 93.64 & 85.56 & 90.77 & 81.25 \\
\hline 8 & S8 & 93.64 & 86.36 & 90.90 & 81.51 \\
\hline 9 & S9 & 94.55 & 85.45 & 90.91 & 81.53 \\
\hline 10 & S10 & 93.64 & 84.55 & 90.31 & 80.34 \\
\hline 11 & $\mathrm{~S} 11$ & 93.64 & 86.36 & 90.58 & 80.87 \\
\hline 12 & S12 & 95.55 & 88.18 & 91.91 & 83.55 \\
\hline 13 & $\mathrm{~S} 13$ & 93.64 & 86.36 & 90.90 & 81.51 \\
\hline 14 & S14 & 93.64 & 86.36 & 91.12 & 81.95 \\
\hline 15 & $\mathrm{~S} 15$ & 94.55 & 87.09 & 90.71 & 81.13 \\
\hline 16 & S16 & 94.55 & 85.56 & 90.77 & 81.25 \\
\hline 17 & S17 & 93.64 & 86.36 & 90.68 & 81.07 \\
\hline 18 & S18 & 93.64 & 85.56 & 90.77 & 81.25 \\
\hline 19 & S19 & 93.64 & 87.27 & 90.82 & 81.35 \\
\hline 20 & S20 & 94.55 & 86.36 & 90.64 & 80.99 \\
\hline
\end{tabular}

The individual recognizing accuracy of subjects was determined using the GUI designed using MATLAB demonstrated in Figure 5.

6.3. Evaluation of Bit Transfer Rate (BTR). The HCI performance can also be evaluated using the BTR. BTR states the number of bits transmitting per unit of time. This criterion includes accuracy and speed in a single value. The BTR for the eleven tasks using convolution features for ERNN and DTDNN are shown in Tables 3 and 4. The bit transfer rates have been determined from the following:

Bit transfer rate $=\frac{60}{T_{\text {act }}}\left[\log _{2} n+p_{a} \log _{2} p_{a}+\left(1-p_{a}\right) \log _{2} \frac{1-p_{a}}{n-1}\right]$.

$n$ indicates the number of eye movements, $T_{\text {act }}$ represents action period, $p_{a}$ specifies the mean accuracy, and 1 $p_{a}$ shows mean recognition error $[42,43]$.
6.3.1. Bit Transfer Rate for Convolution Features Using $E R N N$. The results of the BTR for an individual subject are shown in Table 3 for ERNN using convolution features. Single-trial analysis results of the classifier show that the ERNN has a maximum accuracy for subject 7 and minimum accuracy for subject 13 using single-trial EOG classification for convolution features. The maximum bit rate for the ERNN using convolution features of $83.55 \%$ was obtained for S12 and the minimum bit rate for the ERNN using convolution features of $80.34 \%$ with samples from ten trials which is shown in Figure 6.

6.3.2. Bit Transfer Rate for Convolution Features Using DTDNN. The results of the BTR for an individual subject are shown in Table 4 for DTDNN using convolution features. Single-trial analysis results of the classifier show that the DTDNN has a maximum accuracy for subject 8 and minimum accuracy for subject 13 using single-trial EOG 
TABLE 4: Bit transfer rate of DTDNN using convolution features.

\begin{tabular}{|c|c|c|c|c|c|}
\hline \multirow{2}{*}{ S. no. } & \multirow{2}{*}{ Sub. } & \multicolumn{4}{|c|}{ Bit transfer rate } \\
\hline & & Maximum accuracy & Minimum accuracy & Mean accuracy & BTR accuracy \\
\hline 1 & S1 & 93.64 & 86.36 & 90.41 & 80.53 \\
\hline 2 & S2 & 93.72 & 85.56 & 90.49 & 80.69 \\
\hline 3 & S3 & 93.74 & 86.36 & 90.40 & 80.51 \\
\hline 4 & S4 & 92.74 & 86.36 & 90.58 & 80.87 \\
\hline 5 & S5 & 93.64 & 85.45 & 90.32 & 80.36 \\
\hline 6 & S6 & 93.64 & 85.56 & 90.13 & 79.98 \\
\hline 7 & S7 & 93.64 & 85.45 & 90.59 & 80.89 \\
\hline 8 & S8 & 93.64 & 86.36 & 90.49 & 80.69 \\
\hline 9 & S9 & 93.64 & 85.45 & 90.34 & 80.39 \\
\hline 10 & $\mathrm{~S} 10$ & 93.64 & 86.36 & 90.23 & 80.18 \\
\hline 11 & S11 & 94.55 & 85.56 & 90.47 & 80.65 \\
\hline 12 & $\mathrm{~S} 12$ & 94.55 & 88.18 & 91.76 & 83.24 \\
\hline 13 & S13 & 94.55 & 86.36 & 90.63 & 80.97 \\
\hline 14 & S14 & 94.55 & 85.45 & 90.86 & 81.42 \\
\hline 15 & S15 & 93.64 & 86.36 & 90.50 & 80.71 \\
\hline 16 & S16 & 94.55 & 85.55 & 90.59 & 80.89 \\
\hline 17 & S17 & 93.64 & 86.36 & 90.54 & 80.79 \\
\hline 18 & S18 & 93.64 & 86.36 & 90.59 & 80.89 \\
\hline 19 & S19 & 93.64 & 85.45 & 90.58 & 80.87 \\
\hline 20 & S20 & 94.55 & 86.36 & 90.73 & 81.16 \\
\hline
\end{tabular}

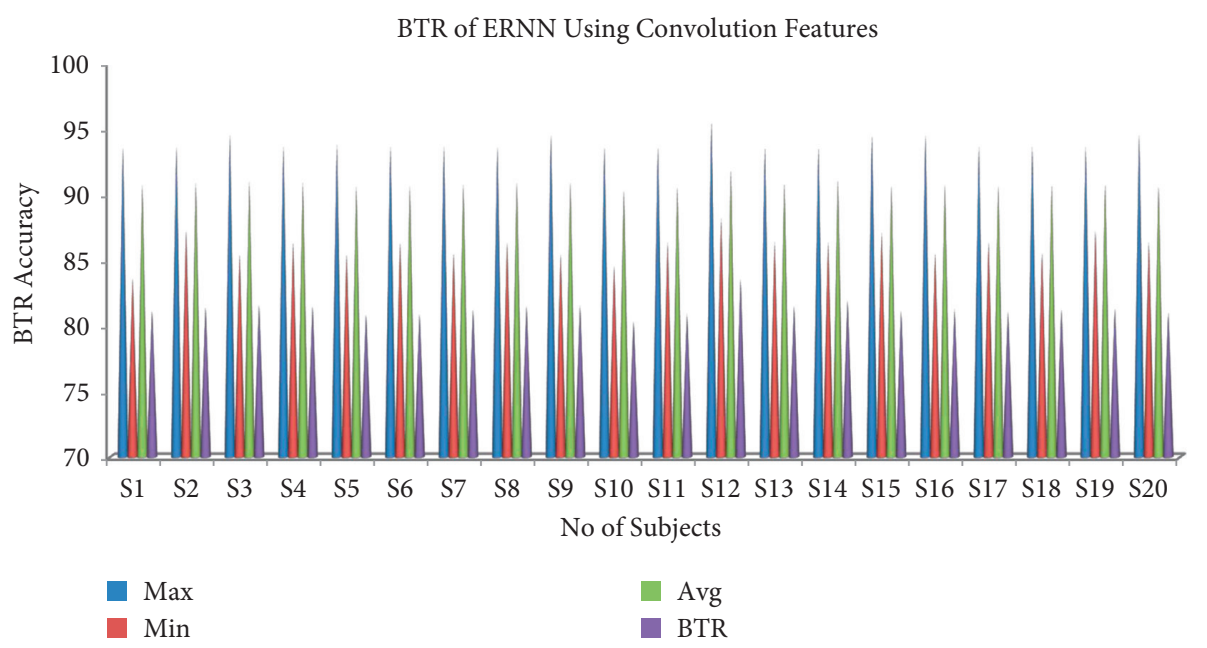

FIGURE 6: Maximum, minimum, and mean accuracy and bit transfer rate for convolution features using ERNN.

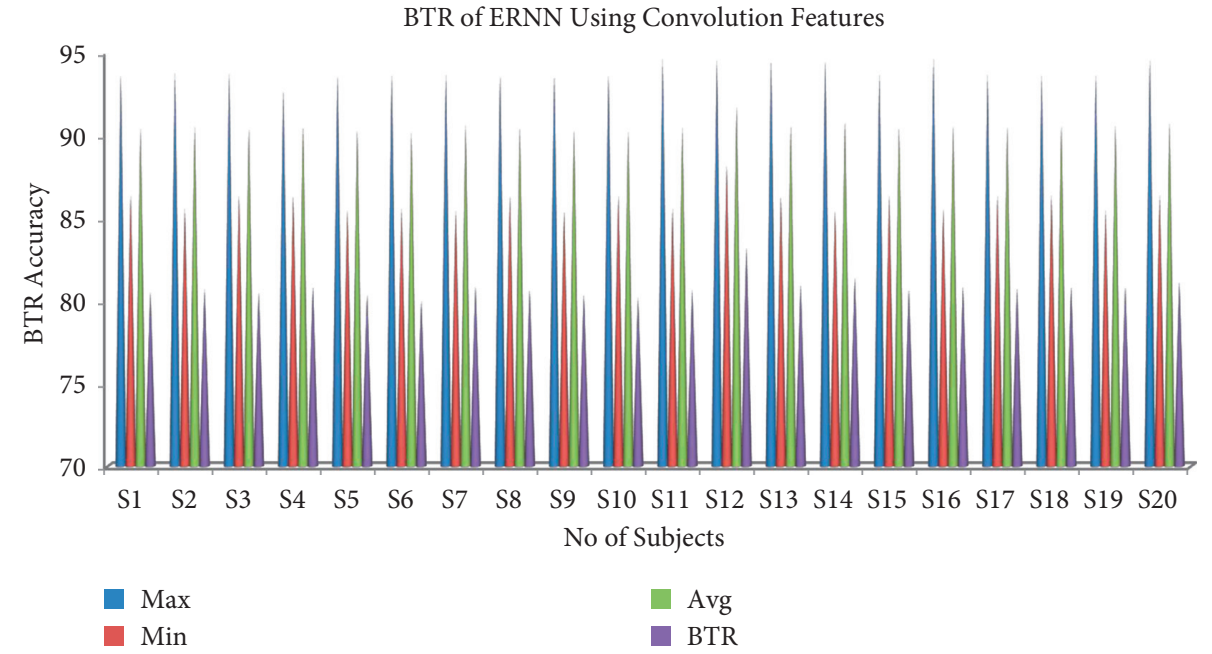

Figure 7: Maximum, minimum, and mean accuracy and bit transfer rate for convolution features using DTDNN. 
classification for convolution features. The maximum bit rate for the DTDNN using convolution features of $83.24 \%$ was obtained for $\mathrm{S} 12$ and the minimum bit rate for the DTDNN using convolution features of $79.98 \%$ with samples from ten trials which is shown in Figure 7.

The accuracy of both network models was correlated based on their accuracy and performance. From the analysis, the ERNN model was significantly higher when related to that of the DTDNN model. From the results, we finally concluded that designing nine-state $\mathrm{HCI}$ is possible by using ERNN model with convolution features.

6.3.3. Limitation of the Study. The system was especially designed for patients with eye movement's activities. The patients without eye movement activities were unable to use this system.

\section{Conclusion}

Eleven tasks were requested to be executed by each individual subject using the ADI T26 Bioamplifier. Two new movements were projected in this research. A new feature extraction algorithm using the convolution theorem has been implemented for extracting features. From the results, it was finalized that the network model using the Elman Recurrent Network model with convolution features was more appropriate for recognizing all the eleven eye movements with the recognizing performance of $90.82 \%$. Single-trial analysis was conducted for an individual subject to analyze the performance of individual subjects. The results show that eye movement classification was subject-oriented. From the bit transfer rate, it was analyzed that the classification performance of convolution features using ERNN was better compared to the DTDNN network model. The experimental analysis proved that the ERNN models were more appropriate for categorizing the collected signals for eleven tasks (both events and nonevents) using convolution features.

\section{Future Study}

Our future extension of this study is to realize online human-machine interaction for nine-state HCI to recognize the high-level human activity in a more efficient way for severely paralyzed persons to fully fill their needs without others' help.

\section{Data Availability}

The data used to support the findings of this study are available from the corresponding author upon request.

\section{Conflicts of Interest}

The authors declare no conflicts of interest.

\section{References}

[1] S. Debener, R. Emkes, M. De Vos, and M. Bleichner, "Unobtrusive ambulatory EEG using a smartphone and flexible printed electrodes around the earflexible printed electrodes around the ear," Scientific Reports, vol. 5, no. 1, Article ID 16743, 2015.

[2] M. de Vos, M. Kroesen, R. Emkes, and S. Debener, "P300 speller BCI with a mobile EEG system: comparison to a traditional amplifierfierJ," Journal of Neural Engineering, vol. 11, no. 3, Article ID 036008, 2014.

[3] S. Debener, F. Minow, R. Emkes, K. Gandras, and M. de Vos, "How about taking a low-cost, small, and wireless EEG for a walk?” Psychophysiology, vol. 49, no. 11, pp. 1617-1621, 2012.

[4] A. B. Usakli, "Improvement of EEG signal acquisition: an electrical aspect for state of the art of front end," Computational Intelligence and Neuroscience, vol. 2010, Article ID 630649, 7 pages, 2010.

[5] S. Makeig, K. Gramann, T. P. Jung, T. J. Sejnowski, and H. Poizner, "Linking brain, mind and behavior," International Journal of Psychophysiology, vol. 73, no. 2, pp. 95-100, 2009.

[6] M. De Vos, K. Gandras, and S. Debener, "Towards a truly mobile auditory brain-computer interface: e," International Journal of Psychophysiology, vol. 91, no. 1, pp. 46-53, 2014.

[7] L. D. Liao, C. Y Chen, I. J Wang et al., "Gaming control using a wearable and wirelesEEG-based brain-computer interface device with novel dry foam-bassensors," Journal of NeuroEngineering and Rehabilitation, vol. 9, no. 5, pp. 1-11, 2012.

[8] C. T. Lin, L. D. Liao, Y. H. Liu, I. J. Wang, B. S. Lin, and J. Y. Chang, "Novel dry polymer foam electrodes for longterm EEG measurement," IEEE Transactions on Biomedical Engineering, vol. 58, no. 5, pp. 1200-1207, 2011.

[9] Y. T. Wang, Y. Wang, and T. P. Jung, "A cell-phone-based brain-computer interface for communication in daily life," Journal of Neural Engineering, vol. 8, no. 2, Article ID 025018, 2011.

[10] A. Campbell, T. Choudhury, S. Hu et al., "Neurophone: brainmobile phone interface using wireless EEG headset," in Proceedings of the 2nd ACM SIGCOMM Workshop Networks,Systems, pp. 3-8, Appl. Mobile Handhelds, New Delhi, India, September2010.

[11] C. Lin and Y. Chen, "Development of wireless brain computer interface with embedded multitask scheduling and its application on real-time driver's drowsiness detection and warning," IEEE Transactions on Biomedical Engineering, vol. 55, no. 5, pp. 1582-1591, 2008.

[12] O. V. Sysoeva, E. B. Lange, A. B. Sorokin, and T. Campbell, "From pre-attentive processes to durable representation: an ERP index visual distraction," International Journal of Psychophysiology, vol. 95, no. 3, pp. 310-321, 2015.

[13] P. P Ghasad, "Design and Implementation of electro-oculogram based brain-computer-interaction," in Proceedings of the International Conference on Computational Intelligence and Communication Networks, Tehri, India, December 2016.

[14] D. Saravanakumar, R. Vishnupriya, and M. R. Reddy, "A novel EOG based synchronous and asynchronous visual keyboard system," in Proceedings of the 2019 IEEE EMBS International Conference on Biomedical \& Health Informatics (BHI), Chicago, IL, USA, May 2019.

[15] R. Barea, L. Boquete, M. Mazo, and E. Lopez, "System for assisted mobility using eye movements based on Electrooculography," IEEE Transactions on Neural Systems and Rehabilitation Engineering, vol. 10, no. 4, pp. 209-218, 2002.

[16] M. Thilagaraj, N. Arunkumar, S. Ramkumar, and S. Hariharasitaraman, "Electrooculogram signal identification for elderly disabled using elman network," Microprocessors and Microsystems, vol. 82, Article ID 103811, 2021.

[17] J. Z. Tsai, C. K. Lee, CM. Wu, J. J. Wu, and K. P. Kao, “A feasibility study of an eye-writing system based on electro- 
oculography," Journal of Medical and Biological Engineering, vol. 28, pp. 39-46, 2008.

[18] S. He and Y. Li, "A single-channel EOG-based speller," IEEE Transactions on Neural Systems and Rehabilitation Engineering, vol. 25, no. 11, pp. 1978-1987, 2017.

[19] J. Ramakrishnan, R. Sivasakthivel, T. Akila, M. Retnadhas, T. T Uthup, and R. Mythily, "Electrooculogram-aided intelligent sensing and high-performance communication control system for massive ALS individuals," The Journal of Supercomputing, vol. 77, 2021.

[20] A. Supratak, H. Dong, C. Wu, and Y. Guo, “DeepSleepNet: a model for automatic sleep stage scoring based on raw singlechannel EEG," IEEE Transactions on Neural Systems and Rehabilitation Engineering, vol. 25, no. 11, pp. 1998-2008, 2017.

[21] Q. T. Obeidat, T. A. Campbell, and J. Kong, "Spelling with a small mobile brain-computer interface in a moving wheelchair," IEEE Transactions on Neural Systems and Rehabilitation Engineering, vol. 25, no. 11, pp. 2169-2179, 2017.

[22] J. Ramakrishnan, D. Mavaluru, R. S. Sakthivel, A. S. Alqahtani, A. Mubarakali, and M. Retnadhas, "Braincomputer interface for amyotrophic lateral sclerosis patients using deep learning network," Neural Computing and Applications, 2020.

[23] X. Xiaoxiao, L. Bin, S. Ramkumar et al., "Electroencephalogram based communication system for locked in state person using mentally spelled tasks with optimized network model," Artificial Intelligence in Medicine, vol. 102, Article ID 101766, 2020.

[24] K. Li and S. Ramkumar, S. Diwakaran, J. Thimmiaraja, and S. Diwakaran, Optimized artificial neural network based performance analysis of wheelchair movement for ALS patients," Artificial Intelligence in Medicine, vol. 102, Article ID 101754, 2020.

[25] A. Dev, M. A. Rahman, and N. Mamun, "Design of an EEGbased brain controlled wheelchair for quadriplegic patients," in Proceedings of the 2018 3rd International Conference for Convergence in Technology (I2CT), pp. 1-5, Pune, India, April 2018.

[26] M. Lokman, A. Dabag, N. Ozkurt, S. Miqdad, and M. Najeeb, "Feature selection and classification of EEG finger movement based on genetic algorithm," in Proceedings of the 2018 Innovations in Intelligent Systems and Applications Conference (ASYU), Adana, Turkey, October 2018.

[27] A. Turnip, D. Soetraprawata, M. Turnip, and E. Joelianto, "EEG-based brain-controlled wheelchair with four different stimuli frequencies," Internetworking Indonesia Journal, vol. 8, no. 1, pp. 65-69, 2016.

[28] M. Z. Ilyas, P. Saad, M. I. Ahmad, and A. R. I. Ghani, "Classification of EEG signals for brain-computer interface applications: performance comparison," in Proceedings of the 2016 International Conference on Robotics, Automation and Sciences (ICORAS), Melaka, Malaysia, November 2016.

[29] S. Ramkumar and C. R. Hema, "Recognition of eye movement electrooculogram signals using dynamic neural networks," KJCS, vol. 6, pp. 12-20, 2013.

[30] C. R. Hema, M. P. Paulraj, and S. Ramkumar, "Classification of eye movements using Electrooculography and neural networks," International Journal of Human Computer Interaction, vol. 5, no. 4, pp. 51-63, 2014.

[31] C. R. Hema, S. Ramkumar, and M. P. Paulraj, "Idendifying eye movements using neural networks for human computer interaction," International Journal of Computers and Applications, vol. 105, no. 8, pp. 18-26, 2014.
[32] S. Ramkumar, K. SatheshKumar, and G. Emayavaramban, "EOG signal classification using neural network for human computer interaction," International Journal of Computer Theory and Applications, vol. 9, no. 24, pp. 223-231, 2016.

[33] K. S. Ramkumar and G. Emayavaramban, "Nine states HCI using electrooculogram and neural networks," International Journal of Engineering \& Technology, vol. 8, no. 6, pp. 30563064, 2017.

[34] S. Ramkumar, K. Sathesh Kumar, and G. Emayavaramban, "A feasibility study on eye movements using electrooculogram based HCI," in Proceedings of the IEEE- International Conference on Intelligent Sustainable Systems, pp. 384-388, Palladam, India, December 2017.

[35] G. Emayavaramban, S. Ramkumar, A. Amudha, and K. Sathesh Kumar, "Classification of hand gestures using FFNN and TDNN networks," International Journal of Pure and Applied Mathematics, vol. 118, no. 8, pp. 27-32, 2018.

[36] C. R. Hema, M. P. Paulraj, R. Nagarajan, S. yaacob, and A. H Adom, "Brain machine interface: analysis of segmented eeg signal classification using short-time pca and recurrent neural networks," Iraq Journal Electrical and Electronic Engineering, vol. 4, no. 1, pp. 77-85, 2008.

[37] J. Gu, S. Ramkumar, G. Emayavaramban et al., "Offline analysis for designing electrooculogram based human computer interface control for paralyzed patients," IEEE Access, vol. 6, Article ID 79151, 2018.

[38] G. Teng, Y. He, H. Zhao, D. Liu, J. Xiao, and S. Ramkumar, "Design and development of human computer interface using electrooculogram with deep learning," Artificial Intelligence in Medicine, vol. 102, Article ID 101765, 2020.

[39] W. Tang, A. Wang, S. Ramkumar, and R. K. R. Nair, "Signal identification system for developing rehabilitative device using deep learning algorithms," Artificial Intelligence in Medicine, vol. 102, Article ID 101755, 2020.

[40] S. Fang, A. F. Hussein, S. Ramkumar, K. S. Dhanalakshmi, and G. Emayavaramban, "Prospects of Electrooculography in human-computer interface based neural rehabilitation for neural repair patients," IEEE Access, vol. 7, Article ID 25506, 2019.

[41] D. L. Hudson and M. E. Cohen, Neural Networks and Artificial Intelligence for Biomedical Engineering, Wiley-IEE Press, New York, NY, USA, 2000.

[42] C. R. Hema, Brain machine interface controlled robot chair, $\mathrm{PhD}$ thesis, Universiti Malaysia Perlis, Malaysia, 2010.

[43] K. Maheswari, S. Ramkumar, K. S. Kumar, P. P. A. Priya, and J. M. A. Navamani, "Offline study for implementing human computer interface for elderly paralyzed patients using Electrooculography and neural networks," International Journal of Intelligent Enterprise, vol. 6, no. 3, pp. 1-321, 2019. 